\title{
Time Frequency Analysis for Blade Rub Detection in Multi Stage Rotor System
}

\author{
Ahmed M.Abdelrhman ${ }^{1, a^{*}}$, M.Salman Leong ${ }^{2, b}$, Yasin.M.Hamdan ${ }^{3, \mathrm{c}}$ \\ and K.H.Hui ${ }^{4}$ d \\ ${ }^{1,3}$ Department of Mechanical Engineering, Faculty of Engineering and Technical Studies, \\ University of Kordofan, Sudan \\ ${ }^{2}$ Institute of Noise and Vibration, Universiti Teknologi Malaysia, Kuala Lumpur, Malaysia \\ ${ }^{3,4}$ RAZAK School of Engineering \& Advanced Technology, Universiti Teknologi Malaysia, Malaysia \\ aahmedrabak@yahoo.com, bsalman@ic.utm.my, 'yassin_ocean@yahoo.com, \\ dkarhoou@yahoo.com
}

Keywords: Blade fault detection, Blade rubbing, Time frequency analysis.

\begin{abstract}
Blade fault is one of the most causes of failure in turbo machinery. This paper discussed the time frequency analysis for blade rubbing detection from casing vibration signal. Feasibility of Short Time Fourier Transform (STFT), Wigner-Ville distribution (WVD) and Choi-Williams distribution (CWD) were examined for blade rub detection in a multi stage blade system through an experimental data. Analysis results showed that these time frequency analysis methods can effectively detect the stage rubbing fault, however, these methods have some inevitable deficiencies in segregating the blade passing frequency (BPF) components of the three rotor stage signals. CWD demonstrated a better time- frequency resolution in analyzing the multi stage rotor system signal.
\end{abstract}

\section{Introduction}

Blades' rubbing is a common fault in turbo-machinery. Barschdorff and Korthauer [1] reported that, blade rubbing is one of the most frequent blade faults in gas turbine and $23 \%$ of total failures in gas turbines contributed by blade rubbing. Blade rubbing can potentially lead to other more destructive failures in machinery such as FOD due to broken blade parts. Blade vibration monitoring and BPF signal analysis can effectively be used for the detection of blade rubbing [2]. For instance, Leong and Lim [84] presented a method using wavelet analysis technique and blade pass frequency signals to detect blade rubbing among other blade faults [3]. Al-Badour, et al. [4] exploits the time frequency analysis (short-time Fourier transforms (STFT) and wavelet transform) for blade-to-stator rubbing detection. Lim and Leong [5] also investigated the use of wavelet analysis for blade faults diagnosis in single stage rotor and they reported that, the time-frequency display could provide a better visualization of the blade conditions and blade rub configuration such as creep rub and eccentricity stage rub could be easily discriminated.

The subject of blade faults diagnosis in multi stage rotor was investigated by the author $[6,7]$, using FFT and wavelet analysis. When blade rubbing occurred, the vibration signal of the multi stage system found to be more complex and containing a number of closely located frequency components other than the fundamental frequencies in the vibration spectrum, and wavelet analysis failed to segregate the BPFs components of the different stages and to identify the location of the stage rub. That was due to the overlapping and interference term.

This work intends to examine the feasibility of the other time-frequency analysis methods, namely, Short Time Fourier Transform (STFT), Wigner-Ville distribution (WVD) and ChoiWilliams distribution (CWD) in blade rubbing detection of multi stage rotor system from casing vibration data. 


\section{Time Frequency Analysis}

Condition monitoring of vibration signal is typically analyzing signals using Fast Fourier Transform (FFT). However, FFT method is lack of time information; in addition to some deficiencies in analyzing the non-stationary signals. Time-frequency distributions such as (Short Time Fourier Transform (STFT), Wigner-Ville distribution (WVD) and Choi-Williams distribution (CWD)), however, offers a wide set of powerful tools to analyze non-stationary signals. In fact, when analyzing signals using FFT based method, the time information is usually encoded in the phase of the FFT and it is simply ignored in the energy spectrum display. Time-frequency analysis is developed to overcome the shortcoming of FFT based methods and to have more informative description of the signals by distributing signals energy contents in a time- frequency display [8].

\section{Short Time Fourier Transform (STFT)}

STFT identified as the windowed Fast Fourier Transform (FFT) magnitude and representation of how the frequency contents of any time signal changes, for each time segment $t$. The STFT distribution is given by Eq. 1:

$$
F_{x}(t, v ; h)=\int_{-\infty}^{\infty} x(u) h^{*}(u-t) e^{-j 2 \pi v u} d u
$$

Where $h(t)$ represents the short time analysis window normally centered around zero and $x(u)$ is the transformed signal. The STFT is a popular machinery diagnostics analysis to run during startup or shutdown and is referred to by the signal analyzer manufacturers as a waterfall function [9].

\section{Wigner-Ville Distribution (WVD)}

WDV is mainly depends on the autocorrelation function when calculating the signal power spectrum, WDV is defined mathematically by Eq. 2 :

$$
W_{x}(t, v)=\int_{-\infty}^{\infty} x\left(t+\frac{\tau}{2}\right) x^{*}(t-\tau / 2) e^{-j 2 \pi v \tau} d \tau
$$

Where $\tau$ is the time lag, and $*$ is representing the complex conjugate of the signal $x$. The WVD distribution fulfills a large number of desirable mathematical properties. In particular, the WVD has been always real-valued; it preserves time and frequency shifts and satisfies the marginal properties.

\section{Choi-Williams Distribution (CWD)}

The CWDD is a distribution that represents the spectral content of the signal in time-frequency plane. The effectiveness of CWD distribution is mainly relying on the nature of the analyzed signal. For instance, if the signal components contain synchronized components in time or in frequency, the Choi-Williams distribution will present strong interferences. Eq.3 bellow defines the ChoiWilliams Distribution:

$$
C_{x}(t, f)=\int_{-\infty}^{\infty} \int_{-\infty}^{\infty} A x(\eta, \tau) \Phi(\eta, \tau) \exp (j 2 \pi(\eta t-\tau f)) d \eta d t
$$

Where:

$A x(\eta, \tau)=\int_{-\infty}^{\infty} x(t+\tau / 2) x^{*}(t-\tau / 2) e^{-j 2 \pi \tau \eta} d \tau$

And the kernel function defined as:

$$
\Phi(\eta, \tau)=\exp \left[-\alpha(\eta \tau)^{2}\right] .
$$




\section{Experimental Study}

Fig. 1 shows the experimental test rig set up. The test rig consisted of three stages of rotor blades each with 8,11 and 13 number of blades, and three stages of stator blade each with 12, 14 and 16 number of blades respectively. The rotor system was driven by an electrical motor via variable speed controller. Blade stage rubbing fault was induced into the test rig at the second stage by attaching small pieces of sheet metal $(0.5 \mathrm{~mm}$ thickness $)$ to all stage two blades to facilitate the rubbing condition against the rotor casing. In this experimental study, two types of signals were measured: Vibration signals measured using data acquisition unit (imc cs-3008) and tri-axial accelerometers mounted on the outer casing of the rotor system at a sampling rate of $2 \mathrm{kHz}, 1024$ sampling frequency, sampling of 100 data points in one complete cycle of shaft rotation of $0.05 \mathrm{sec}$ (for $20 \mathrm{~Hz}$ operating speed) and tachometer phase signal using tachometer installed for speed detection and once per revolution trigger.
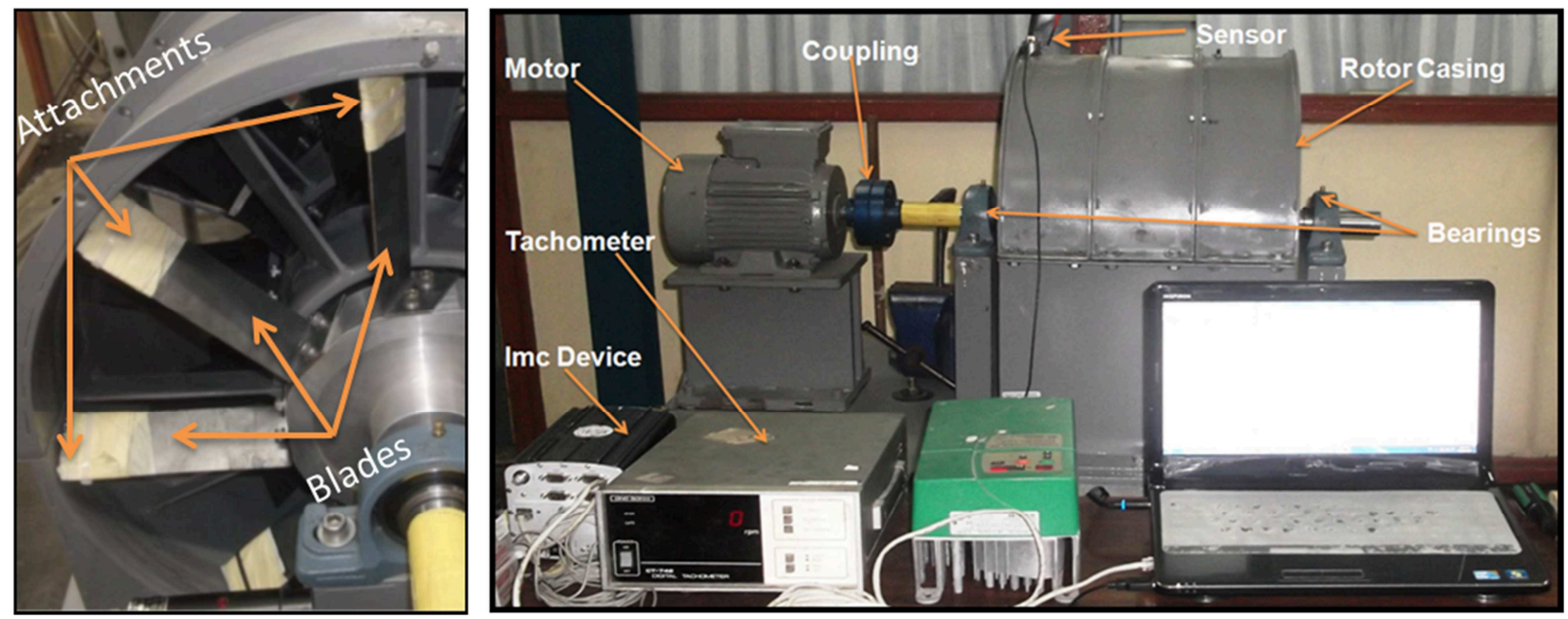

Fig. 1 The experimental test rig set up

\section{Results Analysis and Discussions}

Fig. 2 shows the analyzed time signal, it's FFT and the analysis results of the baseline signal and stage two rubbing signal measured from the rotor casing. SFFT plots were shown that, the symptoms of blades rubbing were evident when compare the faulty signal (b) to the baseline signal (a) and it was demonstrated by the amplitude increment in the faulty signal plot at. It was however, difficult to identify the rubbed blade stage, the location of the rubbing and the number of blades involved. This was deemed due to the insufficient resolutions as al the blade passing frequencies of the three stages were lumped together and overlapped into each other.

Time frequency distribution of WVD showed that, the method can effectively indicate the presence of blade stage rubbing, although, the plot of the rubbing signal (b) and the baseline signal plots (a) were appeared relatively similar to each other, but the coefficient amplitude is increased. It was also noticed that, the energy contents of the signal is well located in the WVD frequency plane, but it was not well distributed in the time plane. WVD demonstrated some advantages in multi stage blade rub detection.

For Choi-Williams distribution, comparing the baseline condition (a) with the result of the stage two induced rubbing condition, visible variations in the rotor CWD map were readily observed and the presence of blade rubbing fault was demonstrated as amplitude decrement. However, the effects of frequency overlapping and interference made the CWD incapable of segmenting the three BPFs components. 

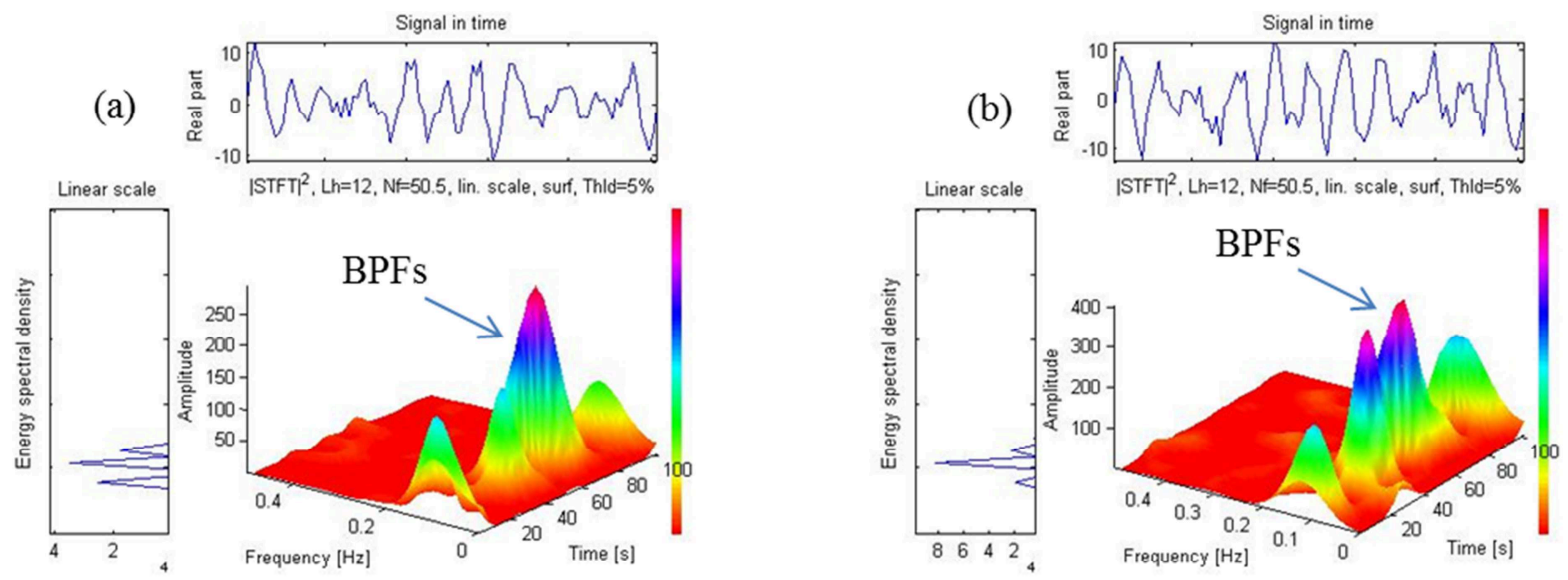

Short Time Fourier Transform

(a)
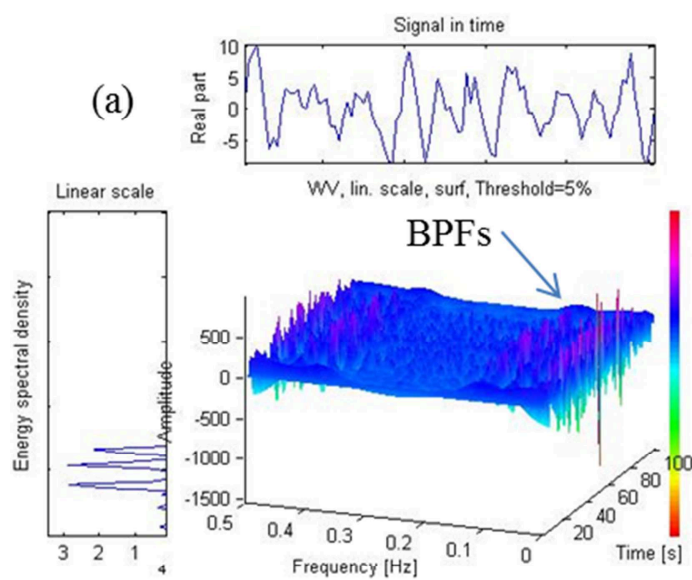

(b)
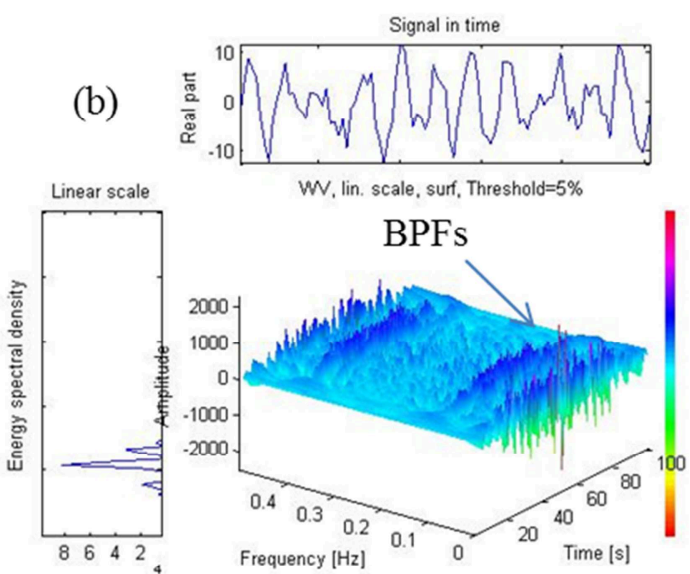

Wigner-Ville distribution

(a)
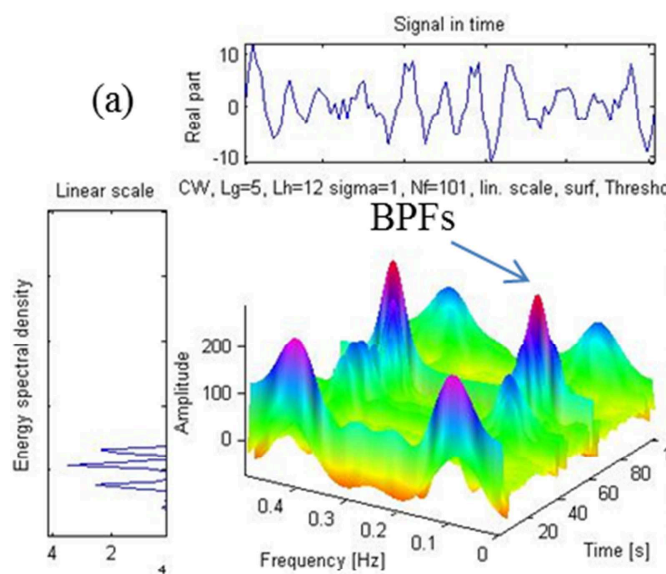

$C W, L g=5, L h=12$ sigma $=1, N=101$, lin. scale, surf, Threshold $=5 \%$

(b)
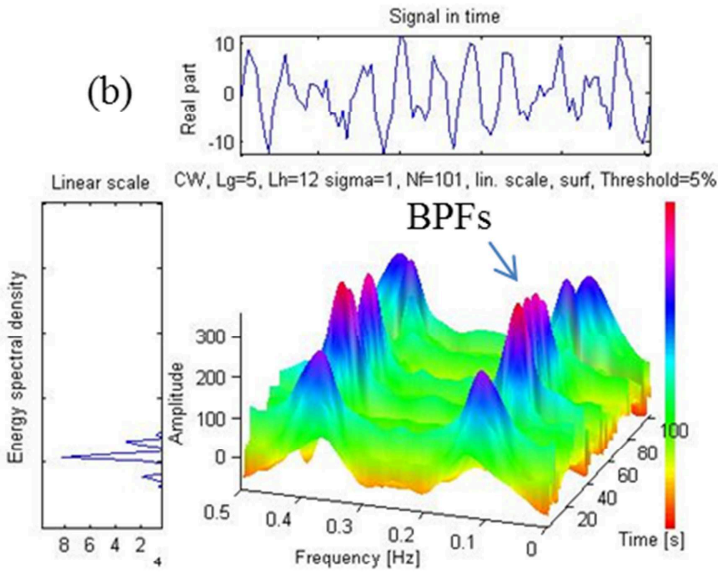

Choi-Williams distribution

(a) Baseline signals

(b) Blade rubbing signals

Fig. 2 Analysis results of SFFT, WVD and CWD, baselines and rubbing conditions

\section{Summary}

The objective of this work was to examine the feasibility of time frequency analysis in blade fault detection in multi stage rotor system, in particular the application of STFT, WDV and CWD in multi stage blades rubbing detection. Signals being used in this study were acquired from rotor test rig developed to simulate the real operational conditions of multi stage rotors. Analysis results of the experimental data showed that, these time frequency analysis could readily detect the blade 
stage rubbing faults based on the different patterns produced by these methods and the faults occurrences demonstrated as amplitudes increments in the computed coefficients. In this instance, these methods can effectively differentiate the faulty condition from the baseline condition. However, STFT, WDV and CWD distributions demonstrated some inevitable deficiencies in segregating the blade passing frequency (BPF) components of the three rotor stage signals and display them separately in the time-frequency plane. Therefore, identifying the exact location of the rubbed blade stage and number of blades involved, were difficult to be identified base on these types of analysis. In this study, CWD found to be more sensitive in blade stage rubbing detection compared to other methods and showed better time-frequency resolution in analyzing the multi stage rotor system signal.

\section{References}

[1] Barschdorff and Korthauer, "Aspects of Failure Diagnosis on Rotating Parts of Turbomachinerys Using Computer Simulation and Pattern Recognition Methods," in International Conference On Condition Monitoring, Brighton, United Kingdom, May 21-23, 1986.

[2] A. M. Abdelrhman, L. M. Hee, M. S. Leong, and S. Al-Obaidi, "Condition Monitoring of Blade in Turbomachinery: A Review," Advances in Mechanical Engineering, vol. 2014, p. 10, 2014.

[3] M. S. Leong and H. Lim Meng, "Blades rubs and looseness detection in gas turbines operational field experience and laboratory study," Journal of Vibroengineering, vol. 15, pp. 13111321, 2013.

[4] F. Al-Badour, L. Cheded, and M. Sunar, "Non-stationary vibration signal analysis of rotating machinery via time-frequency and wavelet techniques," in Information Sciences Signal Processing and their Applications (ISSPA), 2010 10th International Conference on, 2010, pp. 21-24.

[5] M. H. Lim and M. S. Leong, "Improved Blade Fault Diagnosis Using Discrete Blade Passing Energy Packet and Rotor Dynamics Wavelet Analysis," in ASME Turbo Expo Glasgow, UK, 2010, June. , pp. 1-7.

[6] A. M. Abdelrhman, M. S. Leong, L. M. Hee, and W. K. Ngui, "Application of wavelet analysis in blade faults diagnosis for multi-stages rotor system," Applied Mechanics and Materials, vol. 393, pp. 959-964, 2013.

[7] A. M. Abdelrhman, M. S. Leong, L. M. Hee, and K. H. Hui, "Vibration Analysis of Multi Stages Rotor for Blade Faults Diagnosis," Advanced Materials Research, vol. 845, pp. 133-137, 2014.

[8] F. Auger, P. Flandrin, P. Gonçalvès, and O. Lemoine, "Time-frequency toolbox CNRS FranceRice University (1996).".

[9] H. A. Gaberson, "Application of Choi\&\#x2014;Williams Reduced Interference Time Frequency Distribution to Machinery Diagnostics," Shock and Vibration, vol. 2, 1995. 